\title{
MOBILE MAPPING BY FMCW SYNTHETIC APERTURE RADAR OPERATING AT 300 GHZ
}

\author{
S. Palm ${ }^{\mathrm{a}, \mathrm{b} *}$, R. Sommer ${ }^{\mathrm{a}}$, A. Hommes ${ }^{\mathrm{a}}$, N. Pohl ${ }^{\mathrm{a}}$, U. Stilla ${ }^{\mathrm{b}}$ \\ ${ }^{a}$ Fraunhofer FHR, Institute for High Frequency Physics and Radar Techniques, \\ 53343 Wachtberg, Germany - stephan.palm@fhr.fraunhofer.de \\ ${ }^{\mathrm{b}}$ Photogrammetry and Remote Sensing, Technische Universitaet Muenchen, Muenchen, Germany - stilla@tum.de
}

Commission I, WG I/2

KEY WORDS: Mobile Radar Mapping, Car-borne SAR, Street Mapping, Ultra-High Resolution SAR, Subcentimeter Resolution

\begin{abstract}
:
While optical cameras or laser systems are widely used for mobile mapping low attention was payed for radar systems. Due to new semiconductor technologies, compact and leight weight SAR systems based on the Frequency Modulated Continuous Wave (FMCW) principle in the millimeter wave domain can serve for mobile radar mapping on cars. For mapping of long stripes along roads in close range a special strategy for focusing of SAR images was developed. Hereby local adapted planes for processing are used considering the IMU data of the sensor. An experimental system was designed for high resolution radar mapping of urban scenes in close range geometry. This small and leight weighted system has a bandwidth of $30 \mathrm{GHz}(5 \mathrm{~mm}$ resolution) and operates with $300 \mathrm{GHz}$ in the lower terahertz domain. Experiments with a van in an urban scenario were carried out for proof of applicability of an operating SAR system resolving objects in the subcentimeter domain. The results show that narrow cracks in the asphalt of the road are visible and the measuring of small metallic objects placed in the scene is possible. Based on this mobile mapping techniques a first result from an acquisition of vertical facade structure is shown.
\end{abstract}

\section{INTRODUCTION}

Synthetic aperture radar imaging from satellites or airborne platforms is a well established technology for mapping the earth surface. Active illuminating, penetrating clouds and smoke and high resolution indepenent of distance are the advantage of such SAR sensors. While high resolution mobile mapping systems are currently dominated by camera or laser based scanning systems (Ellum and El-Sheimy, 2002, Paparoditis et al., 2012), low attraction was payed for radar systems with high imaging resolution capabilities. Conventional airborne SAR systems operating usually in X- or Ka-band are not practical for the usage of car-borne platforms due to their aperture size - far field condition for close distances - and their limited resolution capacity. There were only few experiments like (Frey et al., 2013) to test for specific conditions of car-borne SAR systems. However, to our knowledge none of them were mapping surface structures at close distance of only several meters in subcentimeter resolution.

In recent years compact and light weight FMCW radar systems in the millimetre and also lower terahertz domain become applicable for mobile mapping scenarios. Operational airborne SAR systems in these frequency domain are currently on the scale of some kilograms or even below (Otten et al., 2014), (Kinghorn and Nejman, 2009). The use of even higher frequencies allow for very small aperture sizes and measurements in near field. Reaching the lower terahertz domain with modern, coherent radar sensors, bandwidths are now available which can resolve down to a few millimeters. The compactness and the resolution of these new sensor generations offer a variety of new potential applications where the use of an imaging radar so far was hardly possible. The deployment of new carrier platforms like cars or small remotely piloted aircraft systems is now possible.

In this paper we want to focus on car-borne high resolution mobile mapping applications and therefore conducted first experi-

\footnotetext{
${ }^{*}$ Corresponding author.
}

ments with a current state experimental radar system which was developed at Fraunhofer FHR. The special street mapping geometry and the close range of the sensor raises new challenges on the focusing geometry. We use locally adapted planes for focusing considering the IMU data of the sensor. Further, these experiments should give first results on processing such high frequencies and bandwidths and to what extend fine-scaled signatures and object textures in mobile mapping scenarios can be resolved by radar. The sensor operates in the lower terahertz domain with an operating frequency of $300 \mathrm{GHz}$ and a bandwidth of $30 \mathrm{GHz}$. With these parameters a theoretical resolution of $5 \mathrm{~mm}$ can be processed. With the SAR sensor mounted on a platform in a moving van, data were collected by driving on conventional roads illuminating the opposite roadway or parking lanes. The SAR system's ability of resolving different metallic objects in size and characteristics down to a radar cross section of $-43 \mathrm{dBsm}$ placed in the scenario was validated. The results are promising that for monitoring runways or roadways, very small metallic objects which may cause a threat are detectable. A few millimeters deep cracks in the asphalt of the road are displayed in the SAR image. In addition, these experiments can serve as groundwork for a future use of such a SAR sensor even on a multicopter.

\section{STREET MAPPING GEOMETRY}

The following methodolgy describes the focusing strategy which is used, if there is no prior information such as a digital surface model (DSM) or digital elevation model (DEM) of the observed area. This is usually the case because of recordings at close range and very high resolution down to a subcentimeter scale. In contrast to image processing of optical sensors, the azimuth focusing process of radar echoes demands a certain foreknowledge of the scene which is being illuminated. The most obvious assumption is that the surface to be imaged is static and does not move during the illumination. Next, the definition of a virtual focusing plane is required, which on the one hand clearly represents the illumi- 
nated surface for as many scenarios. On the other side it should allow an efficient processing of the raw data.

In airborne scenarios the airplane is usually flying some kilometers away from the illuminated scene and without any DSM or DEM there is no information of the scene's topographie. The use of a flat plane model is therefore a straight forward assumption and fits well to airborne scenarios if the region of interest is no challenging terrain. A terrain height change of several meters in the scene is unproblematic compared to the long distance to the sensor.

In theory, this flat plane model works also well for street mapping at close range with the constraint that the streets are almost without gradient. In practical, however, this assumption is unrealistic as streets often show gentle slope or rise. One meter height difference on several hundred meter distance would already be problematic as the sensor is only mounted at close height above the street. The focusing plane might then already be above the sensor configuration. For mapping several hundred meters or even kilometers of streets in close range, this flat plane assumption has to be adapted.

An advantage in the described street mapping geometry, however, is the fact that the sensor is just several meters away from the oberserved area and the car has direct contact to the road surface. Thus, the received GPS/INS data while moving the car measure the terrain information very accurately and densely. With the assumption that the illuminated side of the roadway is at the same height level as the side of the road the car is driving, we extract the GPS/INS data to estimate an instant DEM model. In this configuration the IMU data is not only used for SAR focusing, but also used to calculate the geometry of the instantaneously oberserved scene.

In the following for each synthetic aperture $d \vec{s}$ we assume a flat plane model as defined in Fig.1 with a normal vector $\vec{n}=\left(\begin{array}{l}0 \\ 0 \\ 1\end{array}\right)$ in the cartesian coordinate system.

For small distances the traveled path $\vec{s}$ of the sensor can be approximated to be linear giving the parametrization of

$$
\vec{s}=\vec{r}_{0}+\lambda \vec{a}, \lambda \in \mathbb{R}
$$

with $\vec{a}$ being the approximated linaer directional vector of the sensor's movement and $\vec{r}_{0}$ the start position. The vector $\vec{a}$ can be calculated by the sensor's velocity, position and angular information from the IMU data of the system. It is described by $\vec{a}=\left(d_{\text {east }}, d_{\text {north }}, 0\right)^{\prime}$, with $d_{\text {east }}$ and $d_{\text {north }}$ being the distance travelled in the geographical directions. Determination of the actual sensor position $\vec{r}_{s}(n T)$ solely from GPS information would be far too inaccurate for the focusing process. Assuming a linear motion of the sensor in between each synthetic aperture with the duration of $T$, the calculation of $\vec{r}_{s}(n T)$ can be approximated by an integration or discrete sum of the sensor velocity components to

$$
\begin{aligned}
\vec{r}_{s}(n T) & =\vec{r}_{0}+\int_{0}^{n T}\left(\vec{v}_{\text {east }}(t)+\vec{v}_{\text {north }}(t)+\vec{v}_{\text {down }}(t)\right) t \cdot d t \\
& \approx \vec{r}_{0}+\sum_{m=1}^{m=n}\left(\vec{v}_{\text {east }}(m T)+\vec{v}_{\text {north }}(m T)+\right. \\
+ & \left.\vec{v}_{\text {down }}(m T)\right) \cdot T, \quad m, n \in \mathbb{N}, 0<m \leq n T \quad \text { (2) }
\end{aligned}
$$

with the corresponding sensor velocities $\vec{v}_{\text {east }}(m T), \vec{v}_{\text {north }}(m T)$ and $\vec{v}_{\text {down }}(m T)$ and the sweep duration $T$. As the GPS/INS data rate is much lower than the sweep repetition frequency, a cubic interpolation of the navigational data is used to estimate the different velocities and angular information chirp by chirp.

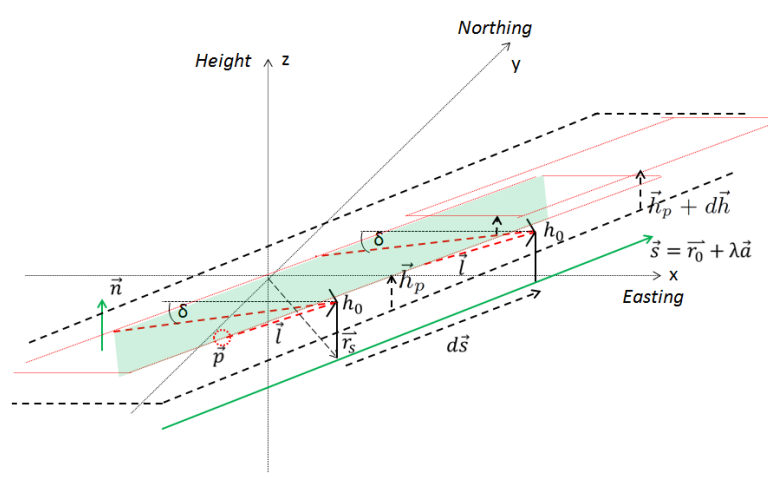

Figure 1: Street mapping geometry for each synthetic aperture $d \vec{s}$. The radar sensor is mounted $h_{0}=2 \mathrm{~m}$ above the street level with an depression angle $\delta$ of 30 degrees. In steps of the length of one synthetic aperture the height of the focusing plane is adjusted by the height distance $d \vec{h}$ calculated from the INS data.

The radar is further mounted on the car's window in a certain height $h_{0} \approx 2 m$ above the ground. The radar beam is constantly illuminating the ground with an adjustable depression angle $\delta$ between $10^{\circ}$ and $40^{\circ}$. The beamwidth of the sensor hereby has an opening angle of up to $20^{\circ}$ in azimuth and range. As the sensor is moving, for each aperture length $|d \vec{s}|$ the level of the actual focusing plain $\vec{h}_{p}$ is constantly adjusted in height, so that it is always at the same distance $h_{0}$ from the sensor. This guarantees the same level of focusing depth and geometry in the case the trajectory is slightly up- or downward rising. With $\vec{l}$ being the actual line of sight of the radar sensor, the intersection with the focusing plane $\vec{p}$ and thus the currently illuminated scene can be calculated with

$$
\vec{p}=\vec{r}_{s}+\frac{\vec{n}^{\prime} \cdot\left(\vec{h}_{p}-\vec{r}_{s}\right)}{\vec{n}^{\prime} \cdot \vec{l}} \cdot \vec{l} .
$$

As small height increases or decreases during a synthetic aperture are compensated in the processing, strong height increases should be avoided as the flat earth model is neglected. Alternating movements of the sensor in the northing or easting directions are unproblematic, as the projection only takes place in the $z$ or height domain.

The azimuth resolution depends on the wavelength of the system and the ability to illuminate and coherently process a given target on the ground in a preferably high angular interval. For sufficiently small angles in the ideal side looking stripmap geometry, the azimuth angular intergration interval $\phi$ can be approximated as

$$
\phi \approx \frac{B W_{\text {Azimuth }}}{f_{c}}
$$

with $B W_{\text {Azimuth }}$ as the desired bandwidth in azimuth domain and $f_{c}$ the carrier frequency. The sensor's radar waveform has a given bandwidth $B W$ of up to $44 \mathrm{GHz}$ and a center frequency $f_{c}$ of $300 \mathrm{GHz}$. With a typical perpendicular distance to the target $r_{\text {mid }}$ of $10 \mathrm{~m}$, this leads to a synthetic aperture length of

$$
|d \vec{s}| \approx r_{m i d} \cdot \tan \frac{\phi}{2} \approx 0.5 m .
$$

Adapting the height level of the focusing plane in this length step 
should be adequate for street mapping.

During the path $d \vec{s}$, the radar signal energy has to be coherently integrated to achieve high crossrange resolution. This demands for stable trajectories of $\frac{\lambda}{10}$ during the pathway. Compared to airborne SAR experiments with X- or Ka-band radar sensors, these aperture length $|d \vec{s}|$ are comparatively very small. With the constraint that at this frequency of $300 \mathrm{GHz}$ a stability of $0.1 \mathrm{~mm}$ is expected, these requirements are apparently at the limit of modern IMU systems. As this high accurancy typically can not be attained during the movement of the sensor in a car, deviations to the ideal trajectory have to be compensated in the signal processing step. This avoids blurring and misplacement effects in the final imaging process.

For the actual focusing of the radar data, a fast time domain approach orientated on the work of (Ribalta, 2011) has been developed. The details of the processing will, however, not discussed in this paper.

\section{SENSOR DESIGN}

The current radar system used for the car-borne experiments is called Millimeterwave Radar using Analog and New Digital Approach (MIRANDA-300). The development of this experimental system is based on the MIRANDA-94 FMCW radar system, which was designed as a compact and light weigth airborne remote sensing SAR at Fraunhofer FHR (Stanko et al., 2011). The sensor can be remotely operated by a person outside the car via a digital data link, but in the described experiments it was operated by the co-driver.

\subsection{Radar Front-End}

The front and back of the radar sensor together with a block diagram of the HF components is shown in Fig. 2. The physical dimensions of the front-end are $20 \times 10 \times 10 \mathrm{~cm}$ with a weight of $\sim 1.5 \mathrm{~kg}$. The used chirp is generated with a signal generator which guarantees a very linear frequency chirp. The signal generator basically depends on a stabilized clock generator working at $916 \mathrm{MHz}$ driving a direct digital synthesizer (DDS). Subsequent to the chirp generator two frequency multipliers are applied. The first one is a by 12 multiplier, increasing the chirp frequency at $8 \mathrm{GHz}$ to $96 \mathrm{GHz}$. Secondly, a by three frequency multiplier is used to achieve the $300 \mathrm{GHz}$ transmit frequency. The resulting bandwidth reaches then $44 \mathrm{GHz}$, giving the system a range resolution of $3.4 \mathrm{~mm}$. This respresents a multiplication factor of 288 . The system needs a normal power supply of $5 \mathrm{~V}$ with a power consumption of approximately $3 \mathrm{~W}$. The final output power is about $5 \mathrm{dBm}$ (Weber et al., 2011, Tessmann et al., 2014). This very low transmission power limits the system applicability to short and mid ranges. A further key component of the system is the fundamental $300 \mathrm{GHz}$ heterodyne receiver module from Fraunhofer IAF (Tessmann et al., 2011). The down-conversion receiver achieves a conversion gain of more than $11 \mathrm{~dB}$ in the frequency range from $270 \mathrm{GHz}$ to $310 \mathrm{GHz}$ with LO-power of only $-12 \mathrm{dBm}$, which is very important for radar applications with low transmit power. In the receiver module the incoming RF-signal is amplified by a four-stage low-noise amplifier (LNA) and subsequently down converted by a single ended resistive HEMT mixer circuit. The excellent flat frequency response of the radar was demonstrated by corner reflector measurements in (Pohl et al., 2015).

\subsection{Radar Back-End}

Real-time SAR capability requires instant access to sampled radar data and continuous information of sensor movements. These

\begin{tabular}{|l|l|}
\hline Frequency & $300 \mathrm{GHz}$ \\
Bandwidth & $44 \mathrm{GHz}$ \\
Sensor Dimensions & $20 \times 10 \times 10 \mathrm{~cm}$ \\
Antenna Dimensions & $4.5 \times 3.5 \mathrm{~mm}$ \\
Weight Front-end & $1.5 \mathrm{~kg}$ \\
Weight SAR System & $5 \mathrm{~kg}$ \\
Power Consumption & $5 \mathrm{~V} / 3 \mathrm{~W}$ \\
Output Power & $5 \mathrm{dBm}$ \\
Noise equivalent $\sigma$ & $-40 \mathrm{~dB}$ \\
\hline
\end{tabular}

Table 1: Sensor Parameter

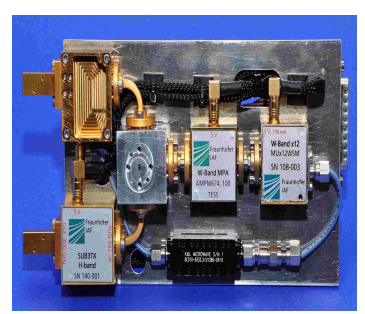

(a)

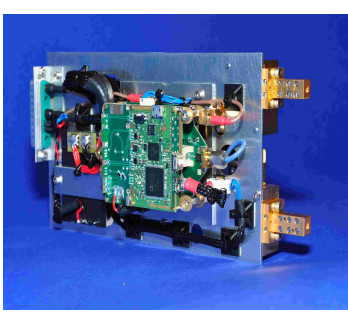

(b)

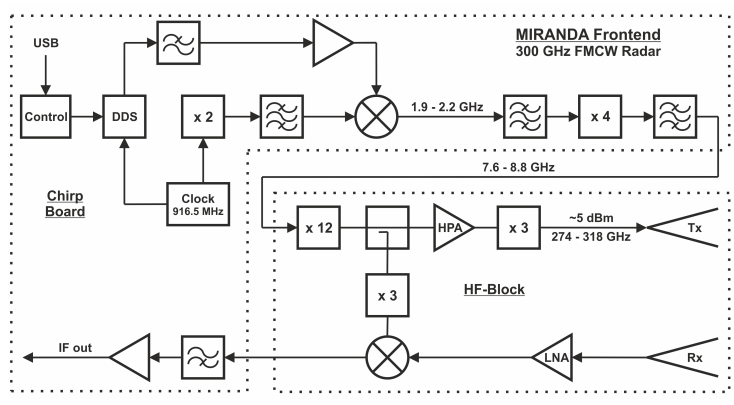

(c)
Figure 2: MIRANDA-300 radar system: (a) Photograph of the high frequency view. (b) Photograph of the chirp generation view. (c) Block diagram of the front-end design. Dimensions are $20 \times 10 \times 10 \mathrm{~cm}$ with a weight of $1.5 \mathrm{~kg}$.

data are measured with an inertial measuring unit with real-time capacity. Due to the FMCW principle, the back-end must sample and record a continously coming data stream of down converted radar echoes and store it disruptively on disk. Data from GPS and from the inertial measuring unit (IMU) are continously received at a different, much lower data rate on a different channel. To achieve a first and fast synchronization of both data sets, both data are processed into one data stream and then transmitted as records.

The incoming GPS/INS packages can further be encoded onto an FM encoded signal on a $5 \mathrm{MHz}$ carrier, which is then down sampled by the second IF input channel of the radar data acquisition unit. This allows the complete signal chain of radar and GPS/INS data to be transmitted via an analog or digital transmission unit. A real-time SAR processing chain can then be started and performed several kilometers away from the SAR sensor. This capability has proven itself especially in the use of airborne systems with payload restrictions (Palm et al., 2014).

\subsection{SAR System}

The radar front-end is integrated in the SAR experimental system shown in Fig. 3. As we need very precise navigational data we use a highly sensitive fiber optic gyro GPS/IMU. A real time interface allows for direct access to the IMU data which enables a 
real time SAR processing chain of the continuous data. Together with the radar front-end the whole SAR system configuration has a weight of less than $5 \mathrm{~kg}$ and can easily be installed inside any car.

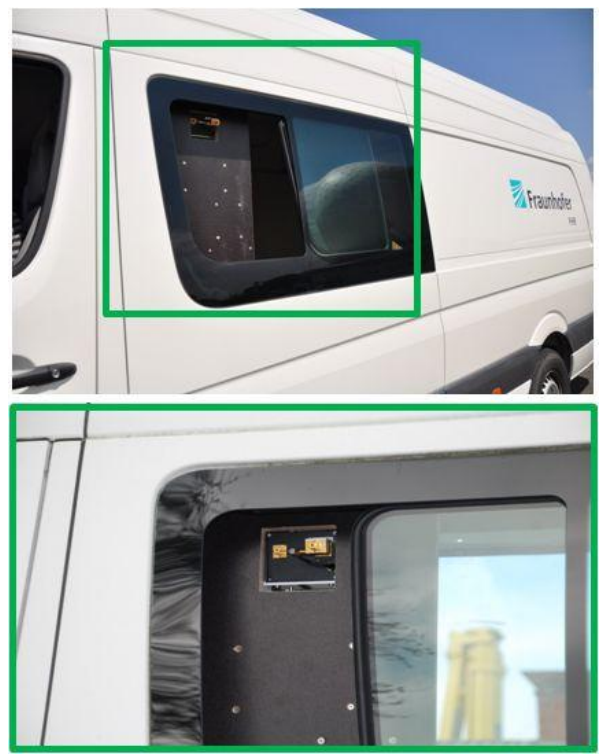

(a)

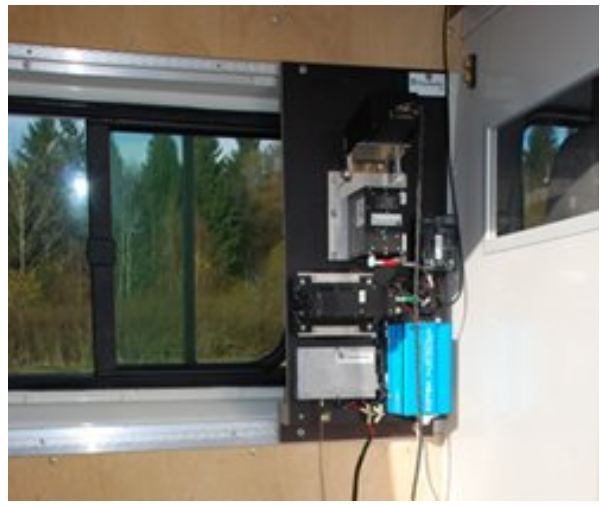

(b)

Figure 3: MIRANDA-300 SAR system: Operation and installation of SAR sensor in van. The radar is mounted on a platform inside the van behind the window. While operating the system the window is opened. The radar is pointing to the left side with a variable depression angle of usually 30 degrees.(a) Exterior of van: Only antennas are visible behind open window. (b) Interior of van: Integration of sensor front- and back-end.

\section{EXPERIMENTS}

The complete Miranda-300 SAR system was mounted on a platform inside a van as shown in Fig.3. It consists of the radar frontend, an RF amplifier, a data recording unit, an IMU and a GPS antenna placed on the top of the van. The transmitting and receiving antennas of the front-end are installed behind the open window of the van in a height of about $2 \mathrm{~m}$ above the street with a variable depression angle of usually 20 to 30 degrees. With this configuration the radar can illuminate a range of $15 \mathrm{~m}$ beneath the moving platform in a side looking geometry. Due to the high bandwidth which has to be handled and the limited data recording, only a stripmap depth of $5 \mathrm{~m}$ in range direction can currently be handled. Further, the limited sweep repetition frequency actually allows the van not to drive faster than $20 \mathrm{~km} / \mathrm{h}$. Otherwise the doppler spectrum of the received echoes is ambiguous. Both constrictions will be reduced significantly by a next generation data recording system which is in the development.

\begin{tabular}{|l|l|}
\hline Sensor Height above Ground & $2 \mathrm{~m}$ \\
Maximum Measuring Distance & $20 \mathrm{~m}$ \\
Measuring Depth & $5 \mathrm{~m}$ \\
Velocity of Van & $20 \mathrm{~km} / \mathrm{h}$ \\
Sweep Repitition Frequency & $5.2 \mathrm{kHz}$ \\
Sweep Duration T & $180 \mu \mathrm{s}$ \\
Processed Bandwidth & $30 \mathrm{GHz}$ \\
Processed Resolution & $5 \mathrm{~mm}$ \\
Pixel Spacing & $2 \mathrm{~mm}$ \\
\hline
\end{tabular}

Table 2: Parameter for SAR processing and mapping geometry

An operator beside the driver or even outside the van can then remotely control the radar system by a laptop and WiFi connection. The range compressed radar data of the current illuminated scene can be watched on the display of the laptop in real time. Together with the navigational data the operator can thus easily intervene in the system if parameters are wrong. We performed several operational tests of the system by driving on conventional roads illuminating the opposite roadway or parking lane. These drives had a length of several hundred meters up to some kilometers. To demonstrate the resolution of very fine-scaled signatures we put several small objects with different characteristics on a parking area. These were different metallic bearing balls ranging from a size of $8 \mathrm{~mm}$ to $22 \mathrm{~mm}$ diameter and various tools and screws. These objects were laid in the center of the scene in a typical distance of $10 \mathrm{~m}$ beneath the van.

\section{RESULTS}

Street distances of up to a few kilometers were mapped in the close range geometry. The height adaption of the focusing plane for each synthetic aperture with a typical length of $0.5 \mathrm{~m}$ was far sufficient for reaching a constant focusing depth of the illuminated streets. The final postprocessed SAR images reach a resolution of $5 \mathrm{~mm}$ in both dimensions. A bandwidth of $30 \mathrm{GHz}(285-$ $315 \mathrm{GHz}$ ) is taken for the range compression of the data. Fig. 4 shows the imaging results for three different streets and parking lanes which were passed by at a distance of $10 \mathrm{~m}$ from the van. The images are sections of longer stripes and show the begin of a street crossing (Fig. 4a), a side verge of a bus lane with striped markings (Fig. 4b) and a parking lane where several experimental objects of different size and characteristics were placed on (Fig. 4c). The strong echo from the curbside as well as the the pattern and the edges of each paving stone is recognissable. The pattern of clean and newly laid paving stones from Fig. 4c appear particularly clear in the SAR images. In contrast, older and covered with sand areas on the left side of Fig. 4a are already less recognizable. The echo of the stone edges is then already lower. Weed in between the paving stone and bounding grasses and plants above the curbside show different characteristics and already foreshortening effects.

Road markings in Fig. 4a and $4 \mathrm{~b}$ are less rough at this frequency and show a more mirror surface than asphalt and therefore stand out clearly from the bright asphalt surface. Even the layout of very detailed objects like gully covers and small cracks of some millimeter size in the asphalt or markings are clearly visible in the SAR images. Fig. 5 and 6 show a roughly $4 \mathrm{~m} \mathrm{x} 4 \mathrm{~m}$ zoom from the center of the image in Fig 4a. Even in this zoom mode the SAR images are still fine resolved. A classification and the 


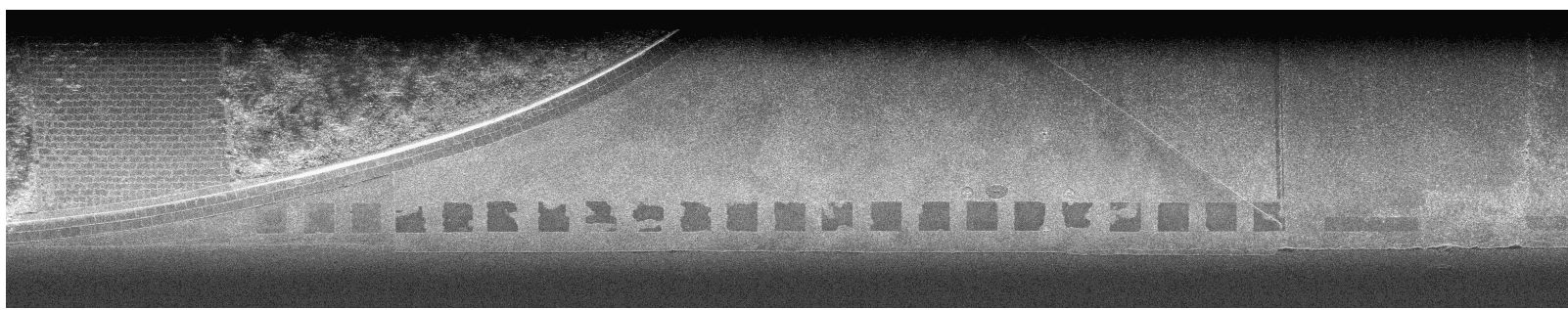

(a)

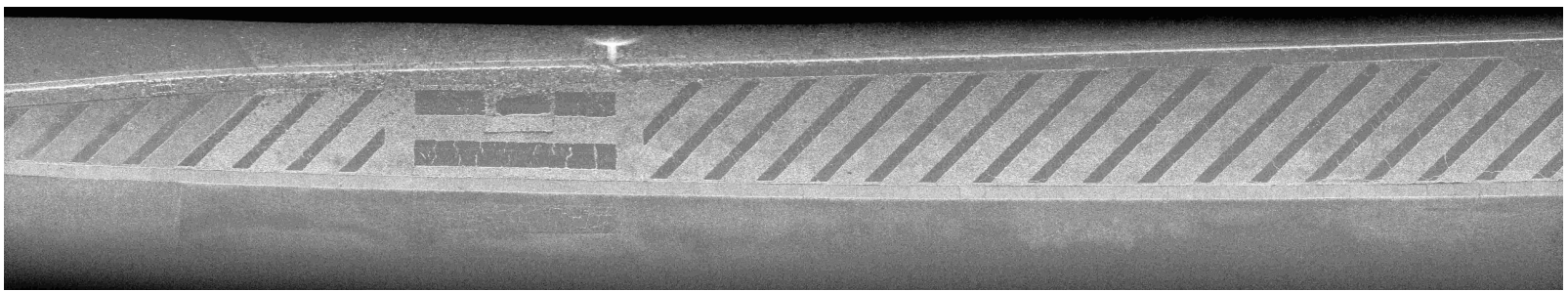

(b)

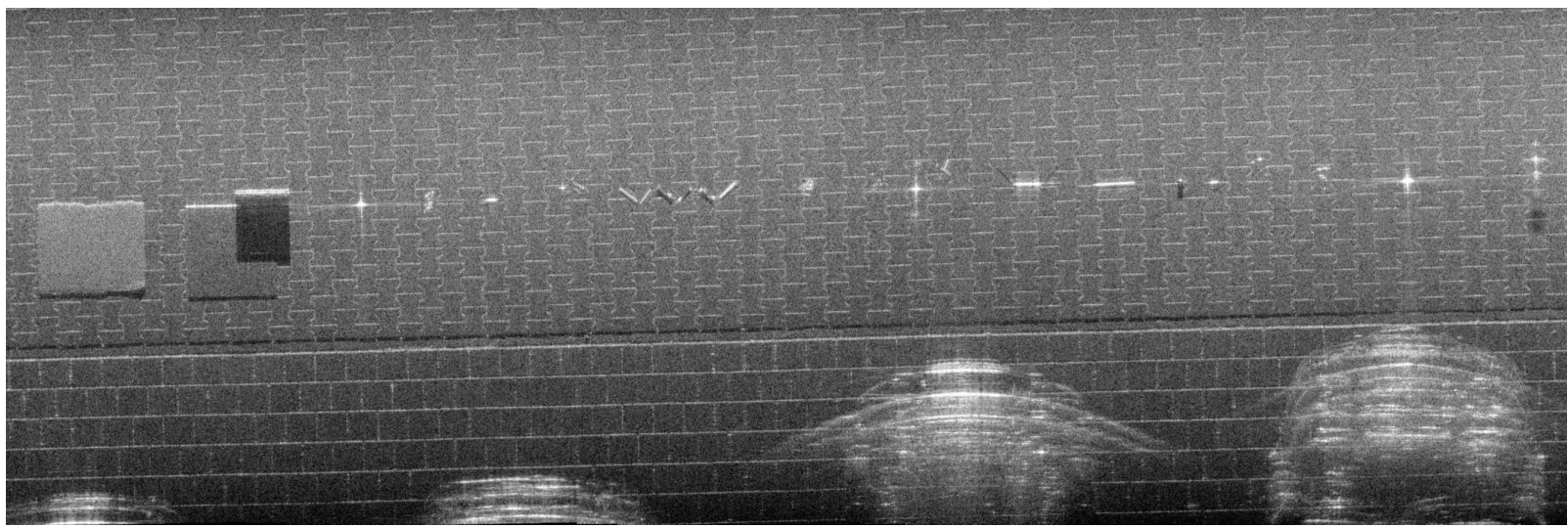

(c)

Figure 4: Street Mapping SAR imaging results: The images are sections of considerably longer strips. The streets and parking lanes were passed by at a distance of $5-10 \mathrm{~m}$ with a velocity of $20 \mathrm{~km} / \mathrm{h}$. The resolution of the SAR images is $5 \mathrm{~mm}$, the stripmap depth about $5 \mathrm{~m}$. (a) Sidewalk (left) and street intersection (right) with road markings (stop marker of priority road) and small cracks in the asphalt. (b) Bus parking lane with striped ground marking. (c) Experimental setup of different metallic objects placed on a parking lane to test for the resolution and penetration capacity of the sensor. On the bottom of the image the front side of parking cars is visible.

orientation of the gully covers can be relatively easily carried out by the viewer. Fraying or missing elements of the white road markings are clearly visible. $2 \mathrm{~cm}$ cracks in the asphalt and holes are identifiable but even finer cracks down to some millimeters are still visuable. A limitation of course is the orientation of the cracks. Best detection is obtained if they are parallel to the sensors trajectory. This guarantees a strong echo from the border of the crack. Asphalt itself, however, has a relatively strong echo resulting in the assumption, that the surface is already rough compared to the used wavelength of $1 \mathrm{~mm}$.

Fig. 4c shows an experimental assembly of different metallic objects placed in the scenario to test for the resolution and penetration capacity of the sensor. On the bottom of the image the front side of parking cars is visible. As one example we want to discuss the objects in the center of Fig. 4c. These are different screws and threaded rods laid into the scene. A zoom of this area is shown in Fig. 7. Again, the pattern of the paving stone and the metallic objects are well recognizable. The possibility of the high zoom mode also allows for measuring objects in the SAR image very accurately which is performed in Fig. 7d. With a defined pixel spacing of $2 \mathrm{~mm}$ the length of the threaded rod can be estimated from the image to $\sqrt{12.48^{2}+6.24^{2}} \mathrm{~cm} \approx 13.95 \mathrm{~cm}$ by simply counting the pixels, see Fig. $7 \mathrm{~d}$. This is very close to the true value of $14 \mathrm{~cm}$ as shown in Fig. 7c. The same is true for the second, slightly smaller object in the scene, which is estimated from the SAR image to be $\approx 11 \mathrm{~cm}$. The validation of very fine scale signatures with metallic bearing balls of $16 \mathrm{~mm}$ diameter and a radar cross section of $-43 \mathrm{dBm}$ has been demonstrated in (Palm et al., 2016). None of the displayed SAR images use autofocus or image enhancements algorithms, which could probably further increase the image quality for subsequent image processing and classification steps.

\section{CONCLUSION}

The quality of the SAR images with a resolution of $5 \mathrm{~mm}$ demonstrates the capability of the used sensor system and clearly shows the potential of radar imaging in the lower terahertz region. The applicability of an operating SAR sensor and the proposed focusing strategy in the street mapping geometry was demonstrated. The compact radar system together with a high performance IMU allows for new possible applications such as mobile radar mapping from car-borne platforms in a yet unknown ultra-high resolution. Therefore, the sensor can supplement the previously used optical sensors in challenging mobile mapping scenarios. The experiments show that for monitoring runways or roadways, very small metallic objects which may cause a threat are detectable and may be classified. The texture of very detailed objects like 


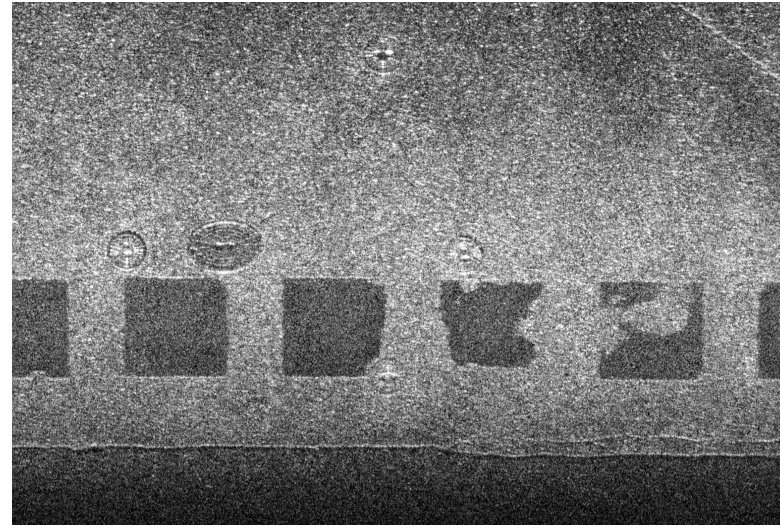

(a)

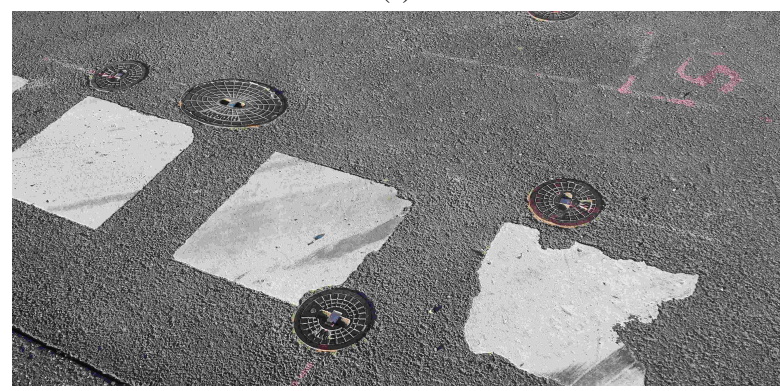

(b)

Figure 5: Zoom (4 m x $4 \mathrm{~m})$ of gully covers and street intersection: (a) SAR image zoom of the center of Fig. 4a. Orientation and texture of the gully covers is clearly observable in the zoom. Also fraying or missing elements of the white road markings and cracks in the asphalt are detectable. (b) Photograph of the scene.

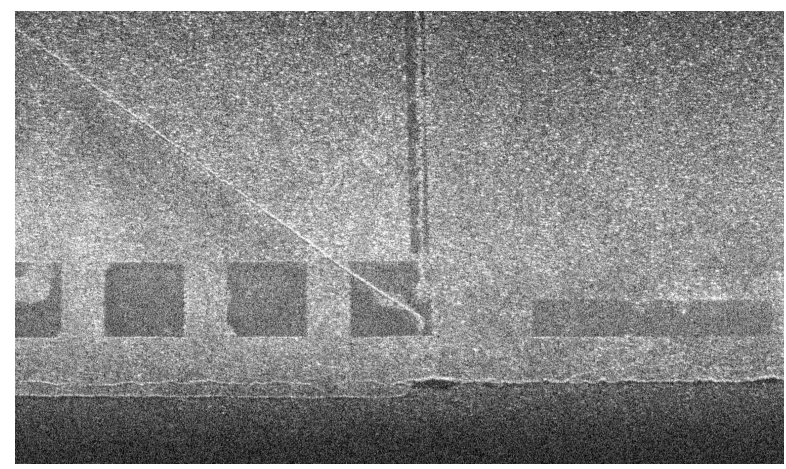

(a)

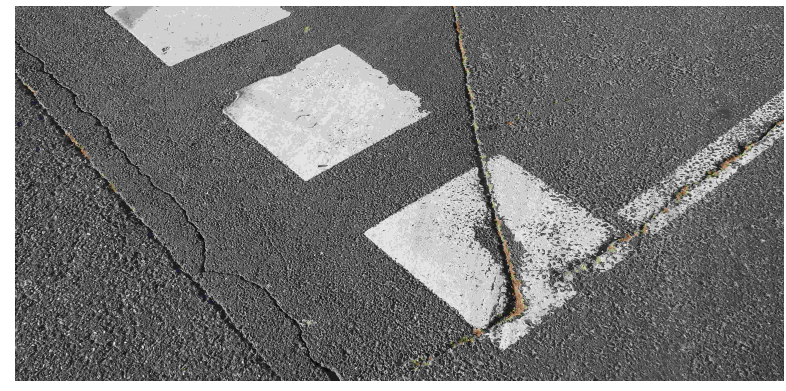

(b)

Figure 6: Zoom (4 m x $4 \mathrm{~m}$ ) of street intersection. (a) SAR image zoom of the center of Fig. 4a. Cracks in the asphalt of $2 \mathrm{~cm}$ down to several millimeters are recognisable. Best detection of cracks, however, if these are parallel to the movement of the sensor. (b) Photograph of the scene.

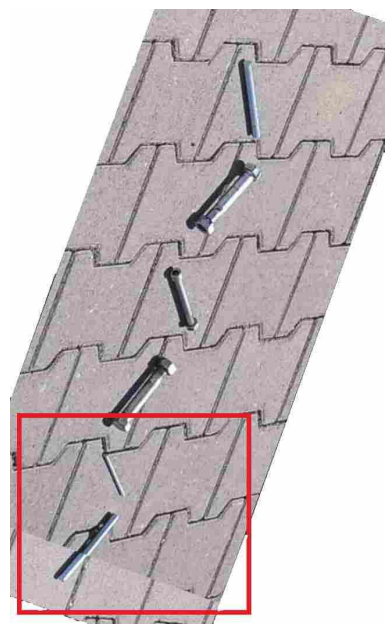

(a)

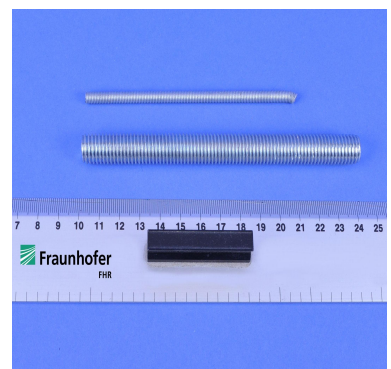

(c)

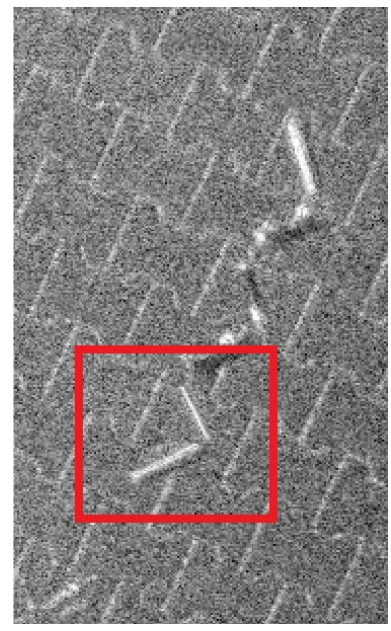

(b)

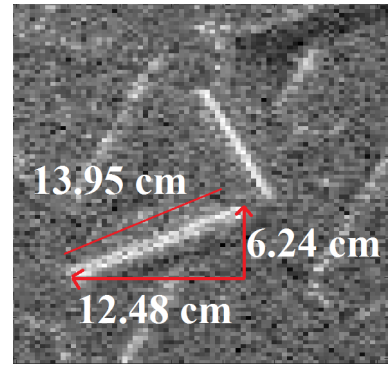

(d)
Figure 7: SAR measurements of metallic objects (screws and threaded rod) on car parking with $5 \mathrm{~mm}$ resolution. Zoom from the center of Fig. 4c. (a) Photograph of the scene. (b) SAR image of these objects on paving stones. (c) Validation of the object's length. (d) Zoom $(\sim 30 \mathrm{~cm} \times 30 \mathrm{~cm})$ of Fig. 7b. Using the defined pixel spacing of $2 \mathrm{~mm}$ the length of the object can be determined to $13.95 \mathrm{~cm}$.

gully covers or narrow cracks in roads are visible and measurable in the SAR images. The use of the continuously received GPS/INS data to directly estimate the height of the illuminated street level works as a straight forward strategy and may be also implemented for a real-time SAR solution.

While performing the campaigns, we experienced considerably higher demands for the quality of the IMU data and the further processing of the data compared to usual airborne campaigns. Due to the high frequency and bandwidth in this domain the high demands of an trajectory estimation in the fraction of millimeters is challenging. We suffer from bad coverage and accessibility of GPS satellites in urban environments. Further start-stop events, which often appear on car-borne systems, are problematic for the quality of the INS data. The use of autofocus algorithms should further enhance the image quality, but has not been implemented so far. Future hardware developments focus on cost effective packaging concepts. Therefore, Fraunhofer IAF is working on advanced RF front-end MMICs which will be mounted in compact and light weigth organic printed circuit boards. This will allow the use of such high resolution SAR sensors even on multicopters in the near future. The problem will then be more likely to meet the high demands on the calculation of the exact flight trajectory in the domain of fractions of a millimeter. 


\section{OUTLOOK}

By a modification of the described geometry in chapter 2 the illumination and SAR processing of vertical structures like house facades or trees in urban areas is a next possible application in the future. First experiments in this directions have already been

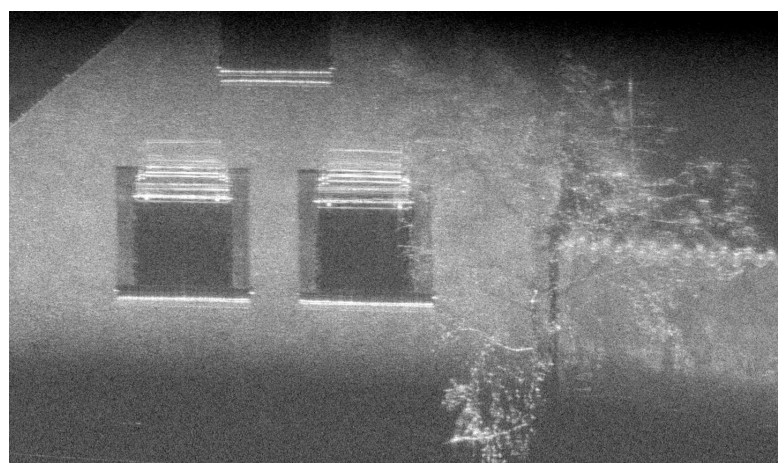

Figure 8: SAR image result of house facade. The sensor is looking upwards with an incidence angle of $\sim 30$ degrees. The first and second floor of a house front with plastered walls is visible. A tree tied to the rain pipe is beneath the right window. Multipath effects and strong echoes from the window benches and roller shutters show strong characteristics. The resolution is $5 \mathrm{~mm}$ with a pixel spacing of $2 \mathrm{~mm}$. The perpendicular distance of the house to the moving radar sensor was about $7 \mathrm{~m}$.

made, where the sensor was turned upwards to illuminate facades from the first level up to the roof. Again, we used the described van and configuration of Fig. 3 and drove down a road in an urban scenario. Fig. 8 shows first results of these experiments. Presented is the first and second floor of a facade with plastered walls. Right of the building is a plastered gateway with a height up to the first level and a completion of the wall consisting of tiles. Placed directly in front of the building and tied to the rain pipe a tree is observable. Due to foreshortening effects the leaves of the tree which are aligned towards the street are closer to the radar sensor and thus appear in short range of the SAR image (bottom). Obviously, the leaves are slightly moving in the wind and therefore show some smearing. The strong echoes from the window region are caused by multipath effects. Due to the fact that the radar sensor is looking upwards, strong echoes are received from window benches and roller shutters as they appear as square edges. Especially the window bench in the second floor shows two strong echos. The first is obviously the edge of the bench aligned towards the sensor, the second echo can be associated to the double bounce of the square edge. The area directly above the benches is located in the radar shadow while the windows appear dark.
Otten, M., Rossum, W. v., Graaf, M. v. d., Vlothuizen, W. and Tan, R., 2014. Multichannel imaging with the AMBER FMCW SAR. In: EUSAR 2014; 10th European Conference on Synthetic Aperture Radar; Proceedings of, pp. 1-4.

Palm, S., Sommer, R., Caris, M., Pohl, N., Tessmann, A. and Stilla, U., 2016. Ultra-High Resolution SAR in Lower Terahertz Domain for Applications in Mobile Mapping. In: The 10th German Microwave Conference (GeMiC), Bochum.

Palm, S., Wahlen, A., Stanko, S., Pohl, N., Wellig, P. and Stilla, U., 2014. Real-time Onboard Processing and Ground Based Monitoring of FMCW-SAR Videos. In: EUSAR 2014; 10th European Conference on Synthetic Aperture Radar; Proceedings of, pp. 1-4.

Paparoditis, N., Papelard, J.-P., Cannelle, B., Devaux, A., Soheilian, B., David, N. and Houzay, E., 2012. Stereopolis II: A multi-purpose and multi-sensor 3D mobile mapping system for street visualisation and 3D metrology. Revue française de photogrammétrie et de télédétection (200), pp. 69-79.

Pohl, N., Stanko, S., Caris, M., Tessmann, A. and Schlechtweg, M., 2015. An ultra-high resolution radar-system operating at 300 GHz. In: Wireless Sensors and Sensor Networks (WiSNet), 2015 IEEE Topical Conference on, pp. 62-64.

Ribalta, A., 2011. Time-Domain Reconstruction Algorithms for FMCW-SAR. Geoscience and Remote Sensing Letters, IEEE 8(3), pp. 396-400.

Stanko, S., Johannes, W., Sommer, R., Wahlen, A., Wilcke, J., Essen, H., Tessmann, A. and Kallfass, I., 2011. SAR with MIRANDA - Millimeterwave Radar using Analog and new digital Approach. In: Radar Conference (EuRAD), 2011 European, pp. 214-217.

Tessmann, A., Leuther, A., Hurm, V., Massler, H., Wagner, S., Kuri, M., Zink, M., Riessle, M., Stulz, H.-P., Schlechtweg, M. and Ambacher, O., 2014. A Broadband 220-320 GHz Medium Power Amplifier Module. In: Compound Semiconductor Integrated Circuit Symposium (CSICs), 2014 IEEE, pp. 1-4.

Tessmann, A., Massler, H., Lewark, U., Wagner, S., Kallfass, I. and Leuther, A., 2011. Fully Integrated $300 \mathrm{GHz}$ Receiver S-MMICs in $50 \mathrm{~nm}$ Metamorphic HEMT Technology. In: Compound Semiconductor Integrated Circuit Symposium (CSICS), 2011 IEEE, pp. 1-4.

Weber, R., Tessmann, A., Zink, M., Kuri, M., Stulz, H.-P., Riessle, M., Massler, H., Maier, T., Leuther, A., Schlechtweg, M. and Kallfass, I., 2011. A W-Band x12 Frequency Multiplier MMIC in Waveguide Package Using Quartz and Ceramic Transitions. In: Compound Semiconductor Integrated Circuit Symposium (CSICS), 2011 IEEE, pp. 1-4.

\section{REFERENCES}

Ellum, C. and El-Sheimy, N., 2002. Land-based mobile mapping systems. Photogrammetric engineering and remote sensing 68(1), pp. 13-17.

Frey, O., Werner, C., Wegmuller, U., Wiesmann, A., Henke, D. and Magnard, C., 2013. A car-borne SAR and InSAR experiment. In: Geoscience and Remote Sensing Symposium (IGARSS), 2013 IEEE International, pp. 93-96.

Kinghorn, A. and Nejman, A., 2009. PicoSAR- an advanced lightweight SAR system. In: Radar Conference, 2009. EuRAD 2009. European, pp. 168-171. 